\title{
MRI as Non-Invasive Modality of Choice for Diagnosis of Cause in Case of Chyluria
}

\author{
Kishor Taori, Amit Disawal, Jawahar Rathod, Rakhi P. Puria, Prasad Wavare, \\ Vishal N. Bakare, Anand Hatgaonkar, Suresh Dhakate \\ Department of Radio-Diagnosis, Government Medical College and Hospital, Nagpur, India \\ Email: kishortaori@gmail.com
}

Received December 6, 2012; revised January 10, 2013; accepted February 5, 2013

\begin{abstract}
Chyluria is a urological manifestation of lymphatic system abnormality as a result of some abnormal communication between these two systems. Although chyluria as such is rare, filariasis is most common cause in Asian countries. This parasitic tropical debilitating disease is known for chronicity, recurrence and resultant complications. This article provides importance of Magnetic Resonance Imaging (MRI), as a non-invasive modality, in the diagnosis of exact etiopathogenesis of chyluria along with clinical symptoms, other investigations required and brief about the management protocols. On MRI, abnormally dilated lymphatics are visualized as meshwork of multiple tubular, tortuous, fluid-filled structures in the retroperitoneum. We report a case of 20 year young adult who presented with history of passing whitish urine on and off since 8 months. MRI helped in the detection of the site and level of lymphatic obstruction and resultant lympho-urinary fistula.
\end{abstract}

Keywords: MRI; Retroperitoneum; Dilated Lymphatics; Chyluria; Filariasis

\section{Introduction}

Chyluria is a condition characterized by passage of milky urine, caused by obstruction or physiological impairment of the renal lymphatics, and passage of lymph into the urinary tract. Chyluria may lead to severe malnourishment due to passage of large quantities of fat and protein in the urine. The exact location of the underlying lympho-urinary fistula is necessary in the management of these cases.

\section{Case Report}

A 20 years male patient came with a history of progressive weakness, recurrent chyluria \& hematuria on and off since 8 months. Initially the patient was investigated with routine blood tests. Serum lipid profile revealed low HDL and near optimal LDL (Table 1).

Biochemical investigations of urine revealed increased triglyceride ( $222 \mathrm{mg} / \mathrm{dl}$, normal $<150 \mathrm{mg} / \mathrm{dl}$ ), Urine total protein $(2.2 \mathrm{gm} / \mathrm{dl})$, Urine albumin $(1.1 \mathrm{gm} / \mathrm{dl})$ level. Based on this baseline investigations, the diagnosis of chyluria was confirmed and further CT Abdomen was done. It revealed multiple small non enhancing hypodensities in retroperitoneum \& pelvis which was misdiagnosed as enlarged lymph nodes with central necrosis with underlying etiology being tuberculosis (Figure 1). The CT scan was again reviewed with clinical symptomato- logy of the patient keeping in mind, but with no positive results. Antitubercular drugs were started, but there was no relief in symptoms, even after 6 months. MRI was advised which showed multiple non enhancing tubular, tortuous, fluid-filled structures in the retroperitoneum (Figures 2-4), which were extending upto left renal hilum (Figures 5-7) on $\mathrm{T} 1 \mathrm{~W}$ fat suppressed sequences suggesting dilated lymphatics with communication to left renal pelvis. The thoracic duct (Figure 8) \& cisterna chili, however showed normal caliber. On imaging the urinary bladder, there were fat-fluid levels noted (Figures 9(a) and (b)). MRI thus demonstrated the exact cause of chyluria in this case, being pyelo-lymphatics fistula with lymph excreted in bladder. As India is an endemic country for filsariasis, it was considered the first differential

Table 1. Lipid profile of the patient.

\begin{tabular}{ccc}
\hline Serum lipid profile & Value & Normal range \\
\hline Cholesterol & $175 \mathrm{mg} / \mathrm{dL}$ & $<200 \mathrm{mg} / \mathrm{dL}$ \\
VLDL & $21 \mathrm{mg} / \mathrm{dL}$ & $2-30 \mathrm{mg} / \mathrm{dL}$ \\
HDL & $33 \mathrm{mg} / \mathrm{dL}$ & $>60 \mathrm{mg} / \mathrm{dL}$ \\
LDL & $125 \mathrm{mg} / \mathrm{dL}$ & $<100 \mathrm{mg} / \mathrm{dL}$ \\
Triglyceride & $106 \mathrm{mg} / \mathrm{dL}$ & $<150 \mathrm{mg} / \mathrm{dL}$ \\
\hline
\end{tabular}




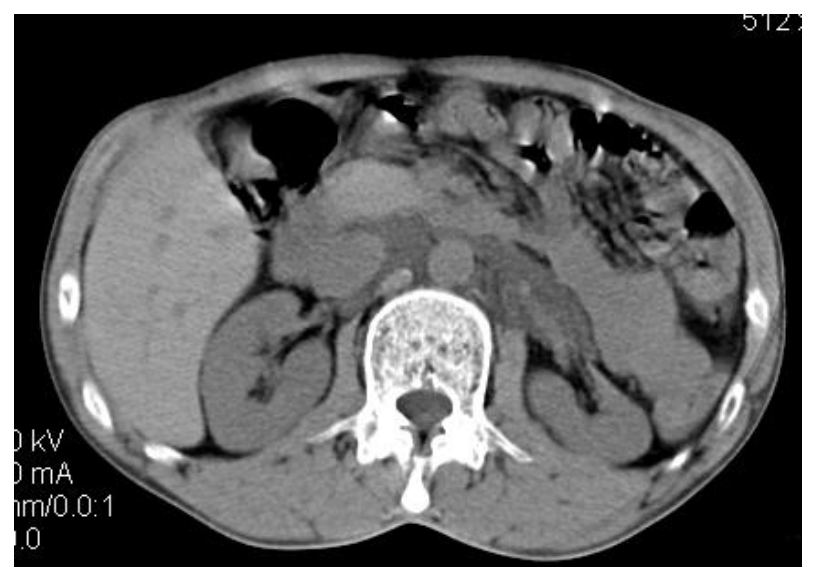

Figure 1. Multiple non enhancing hypodensities in the retroperitoneum encasing aorta \& IVC misdiagnosed as retroperitoneal lymphadenopathy.

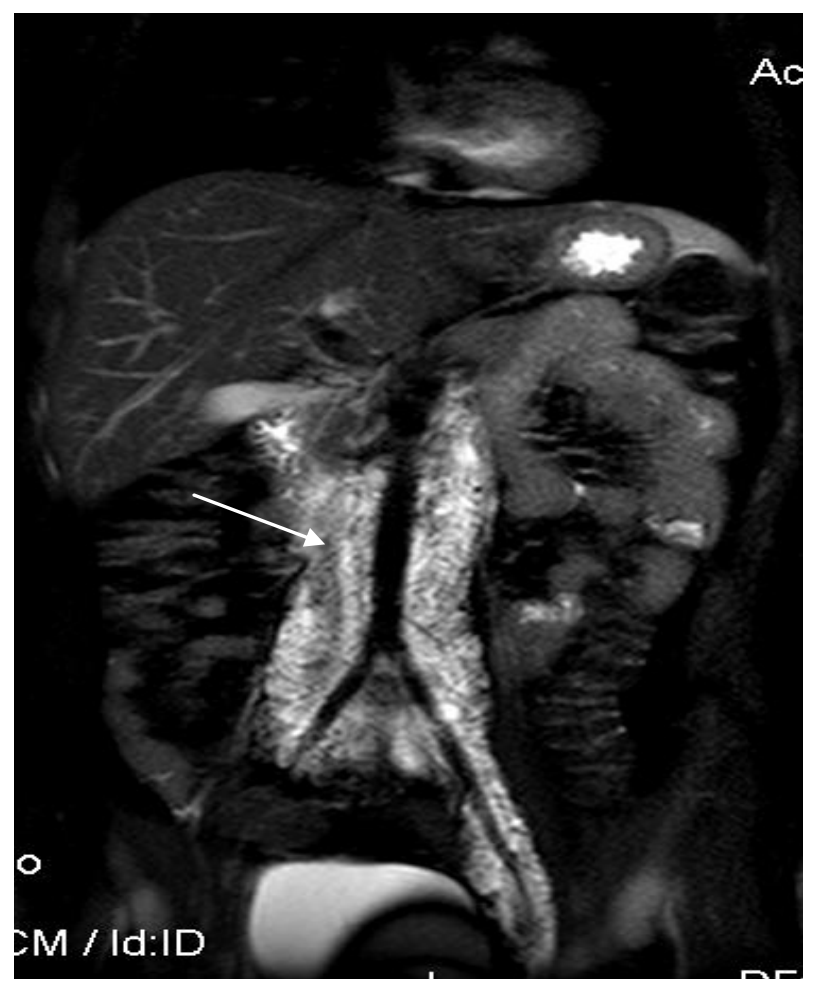

Figure 2. T2W Fat suppressed coronal image showing multiple hyperintense abdomino-pelvic tubular channels encasing aorta, IVC \& its bifurcation s/o dilated lymphatics.

diagnosis of the underlying etiology. Therefore, patient was managed by fat restricted diet \& diethylcarbamazine tablets. On regular follow up, patient is symptomatically relieved.

\section{Discussion}

According to review of literature, there are two theories for the cause of chyluria:

Obstructive theory (Aye and Aung, 1975): Inflamma-

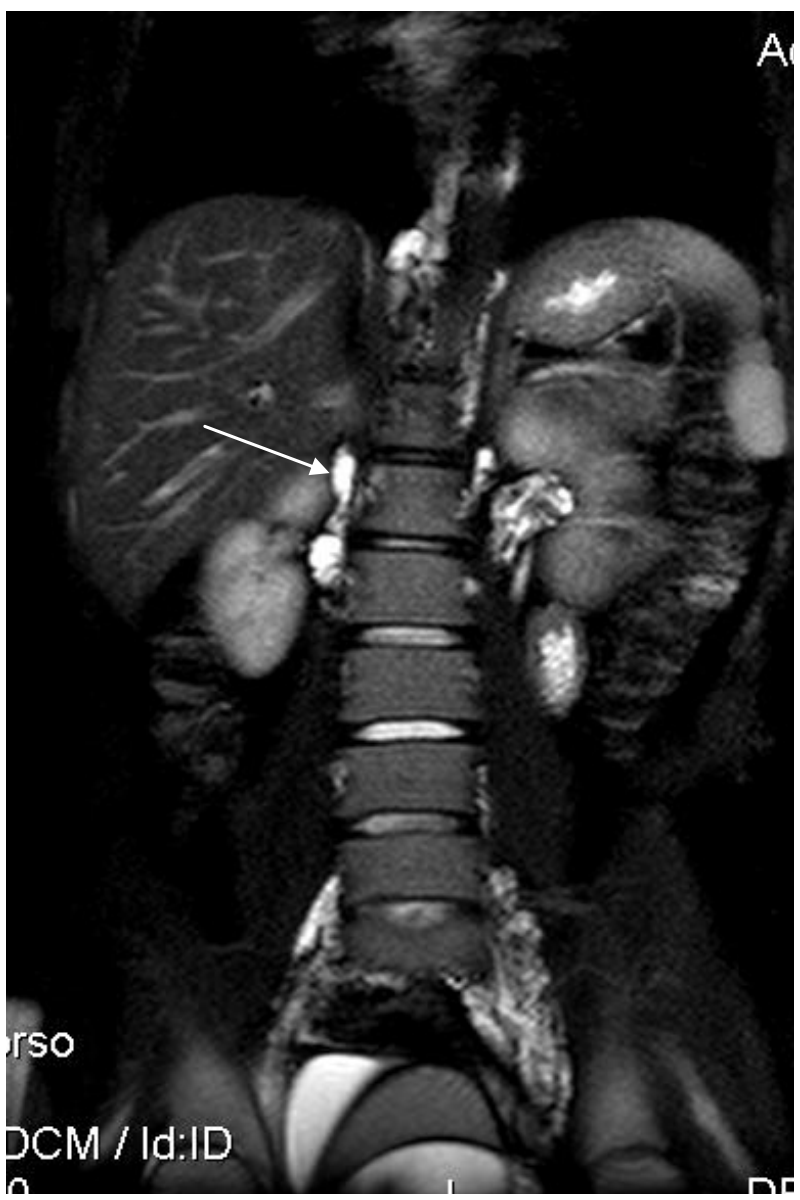

Figure 3. T2W coronal image showing multiple tubular (dilated lymphatics) hyperintensities in pelvic region encasing iliac vessels.

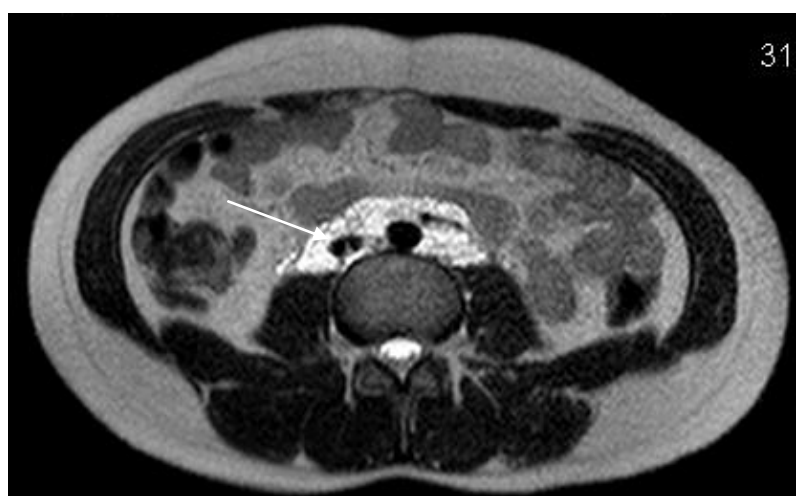

Figure 4. Axial T2W image showing multiple tubular (dilated lymphatics) hyperintensities encasing aorta \& IVC.

tory reactions due to parasitic infestation lead to obliterative lymphangitis, lymphatic hypertension, valvular incompetence and retrograde flow of chyle producing a lymphatico-renal shunt [1].

Regurgitative theory (Ngan and Leong, 1977): Toxic metabolites or immune reaction to parasitic infestation leads to lymphatic ectasia and varicosities, that rupture, 


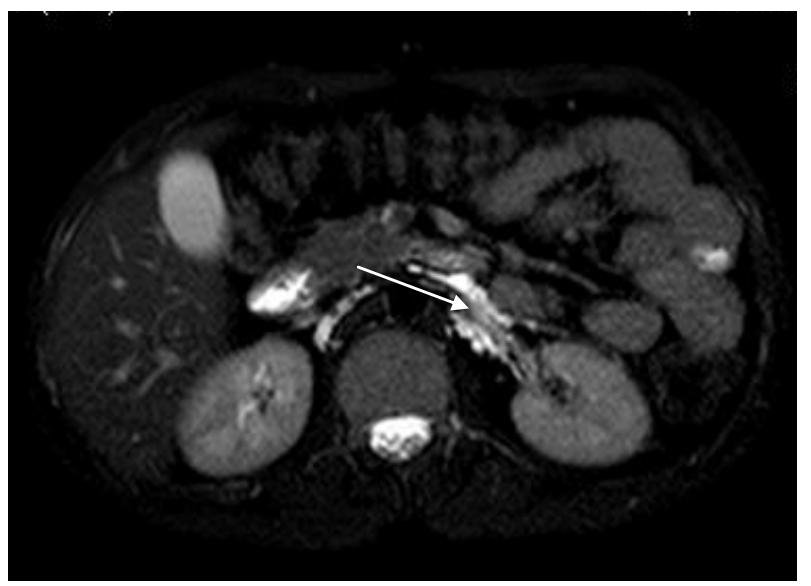

Figure 5. Axial T2W fat suppressed image showing dilated tubular channels extending upto left renal hilum.

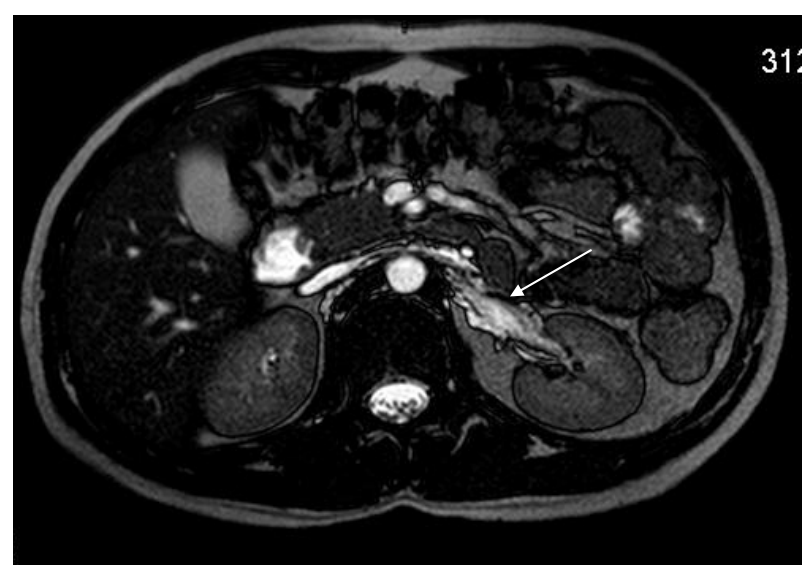

Figure 6. Axial T2W image shows hyperintense tubular channels extending upto left renal pelvis.

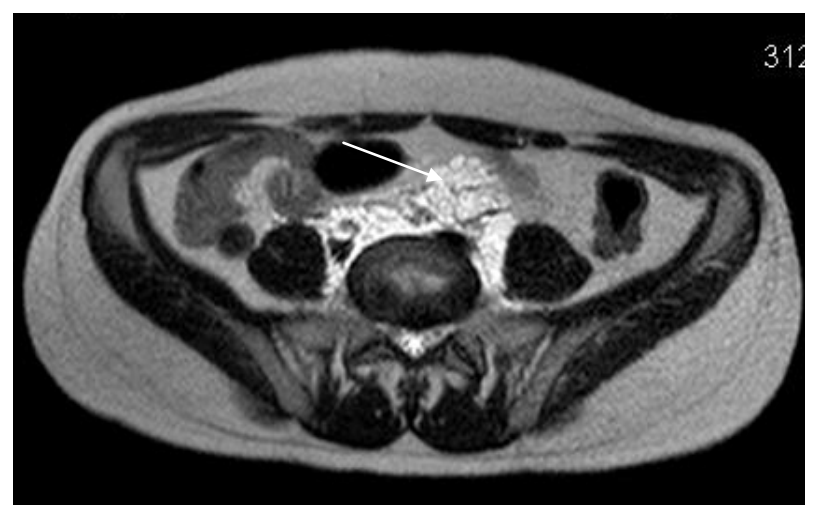

Figure 7. MRI image showing multiple hyperintense tubular channels in pelvic region encasing iliac vessels.

releasing chyle from dilated intestinal lymphatics into the renal system [2].

Chyluria is due to abnormal retrograde or lateral flow of lymph from the intestinal lymphatics to the kidney, ureter or bladder, through way of some abnormal communication, allowing chylous material to be discharged

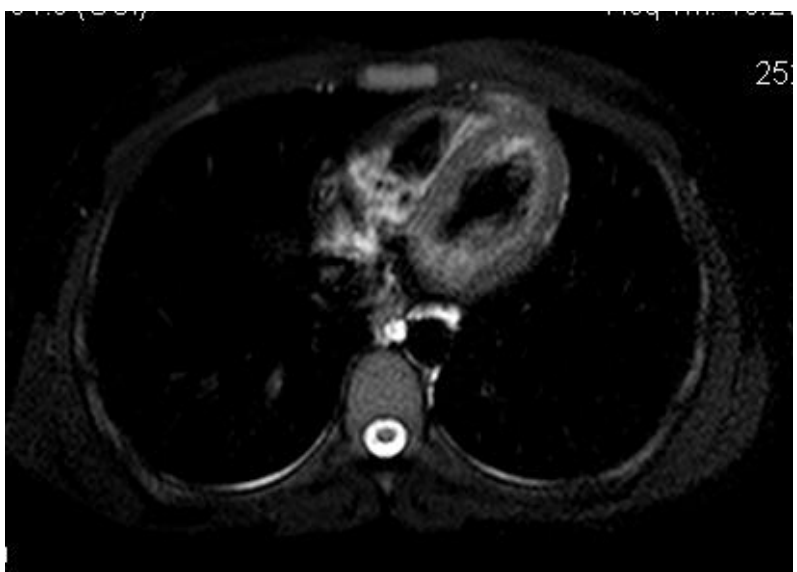

Figure 8. Axial MRI image showing no abnormal dilatation of the thoracic duct.

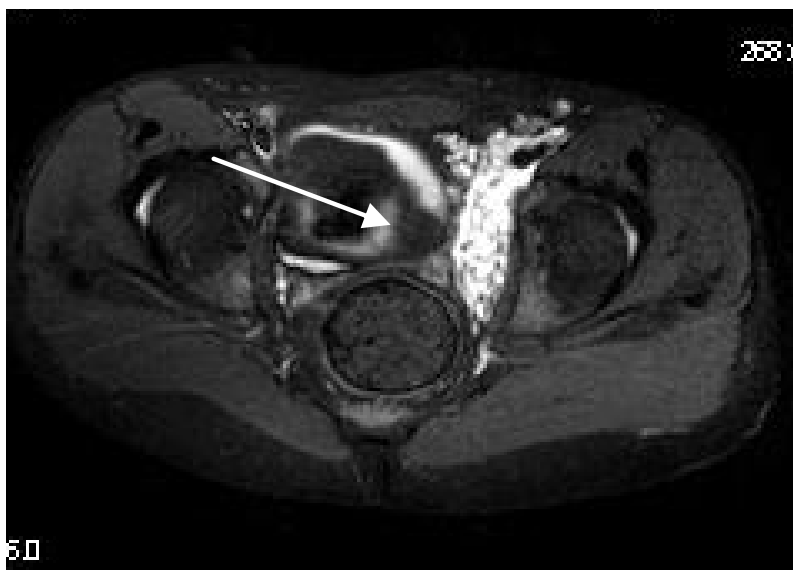

(a)

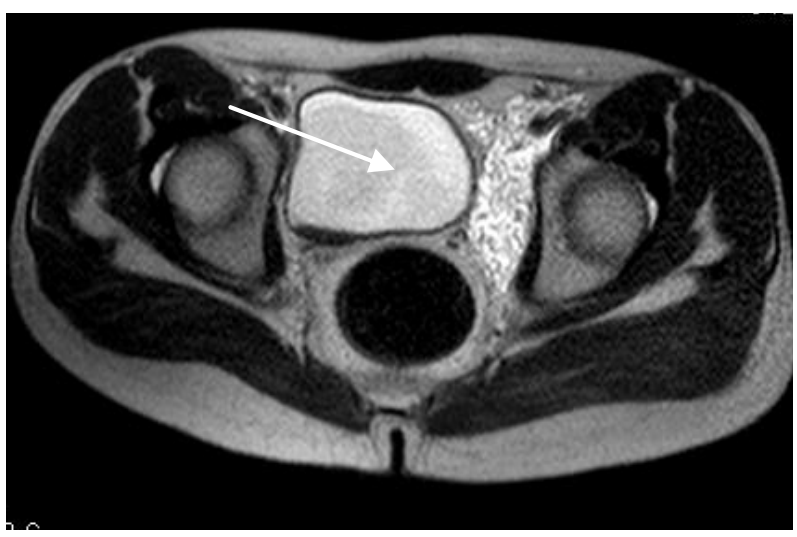

(b)

Figure 9. Altered signal inside the lumen of urinary bladder with signal suppression on fat suppressed sequence s/o presence of fat along with urine.

into the urinary collecting system [3]. Various causative factors have been implicated. However, the most common cause being filariasis in Tropical countries. Passage of chyle into the urine is possibly due to rupture of the lymphatic varices leading to the aperture of one or more 
perirenal lymphatic vessels to the pyelocaliceal system, characterized by milky urine, rich in protein, lipids, cholesterol and triglycerides [4]. This sort of fistula can follow a very chronic course.

On anatomical basis, the renal lymphatics follow the renal vein and end in lateral aortic glands; efferents from renal lymphatics flow to the lumbar trunks.

The intestinal trunks receive lymph from stomach, pancreas, intestine, spleen and liver. Physiologically lymph travels from the lacteals to the cisterna chyli or thoracic duct. Pathological obstruction and/or insufficiency of the valvular system of lymph channels leads to retrograde flow to lumbar lymph glands draining renal lymphatics. Thus there is a short circuiting of chyle drainage from intestinal lacteals to renal lymphatics [4].

\section{Clinical Symptoms}

The most common clinical symptom is passage of milky urine in up to $70 \%$ cases [5]. Other symptoms include chylous clot, bloody and milky urine, dysuria, weakness, frequency, urgency and urinary retention in decreasing order of presentation. The disease is significantly higher in males (86\%) than in females (14\%) [6]. It has been more frequently reported on the left side [7]. Majority of the cases present in the second and third decade of life.

\section{Investigations}

1) Biochemical tests of Urine: If urinary triglycerides are present in morning sample, it is $100 \%$ sensitive and specific for chyluria. Postprandial urine lipids, especially triglycerides ranging from 10 to $1955 \mathrm{mg} / \mathrm{dl}$, can be used as marker for the clinical evaluation of chyluria. Increased protein levels can also be found in urine.

2) Ultrasonography: Floating hyper-echoic component due to the chylous component with anechoic urine in the bladder can be seen.

3) CT scan: Dilated lymphatic channels can mimic a mass of confluent low-density lymph nodes [8]. Fat in the bladder secondary to chyluria can be picked up on CT by differential HU of fluid \& fat.

4) MR scan: It is non-invasive and less time consuming study-best to delineate anatomy (but no functional assessment). On MR urography, the lymphatic channels are visualised as a meshwork of multiple tubular, tortuous, fluid filled structures in the retroperitoneum of the abdomen and pelvis encasing the aorta \& IVC. On axial T1W images, these channels are seen as numerous, small \& nodular hypointensities and as a cloak of diffuse homogenous hyperintensity on T2W axial images [8]. The usefulness of MRI is being further evaluated.

There are other investigations like Lymphangiography \& Lymphangioscintigraphy. Lymphangiography is not routinely done as it is time consuming, invasive \& tech- nically demanding.

\section{Management}

\section{Non Surgical Management}

1) Dietary modifications: A fat restricted diet is recommended ( $<25$ g/day) as absorption of dietary fat leads to chyle formation. Fats containing Medium Chain Triglycerides ( $<12 \mathrm{C}$ atoms) are absorbed directly through portal vessels hence use of coconut oil is recommended [9].

2) Diethyl carbamazine (DEC/Hetrazan/Benocide): The dose is $6 \mathrm{mg} / \mathrm{kg}$ in 3 divided doses after food for 10 - 14 days [10]. Ivermectin $400 \mathrm{mg} / \mathrm{kg}$ (single dose) and/or albendazole $400 \mathrm{mg}$ may be further added with DEC as symptomatic treatment where filarial infection seems to be the cause.

3) Supportive treatment: High protein diet is advised to make up for the protein lost in the form of chyle. Hematinics, multivitamins and green leafy vegetables are recommended in hematochyluria. Bed rest, anti-inflammatory, antipyretics and analgesics are useful in managing lymphadenitis. During acute attacks of chyluria, abdominal binders may be applied to reduce loss of chyle. Cytoscopic bladder wash can be given in cases with clot/ chylous urine retention and/or recurrent urinary tract infections [4].

4) Sclerotherapy: It comprises of instillation of sclerosing agents in renal pelvis \& causes relief of symptom by chemical lymphangitis [4]. The chemicals which can be used are silver nitrate $(0.1 \%-3.0 \%), 0.2 \%$ povidone iodine, $10 \%$ - 25\% potassium iodide, $15 \%$ - 25\% sodium iodide, $50 \%$ dextrose, $76 \%$ hypertonic saline or combination therapy using 5/6 Fureteric catheter after localizing site on cystoscopy in cases of failure of conservative management.

5) Lympho-venous disconnection: It is treatment of choice in severe form of chyluria. A fatty diet 24 - $38 \mathrm{hr}$ prior to surgery facilitates detection of lymphatics. Renolymphatic disconnection operation is performed. The kidney is separated, lymphatics in hilar area coursing along the renal vein are dissected, ligated \& cut. Connective tissue containing dilated lymphatics in the perirenal and hilar region travelling to the kidney are dissected and divided between ligatures-thus stripping these structures completely. Hilar stripping is continued up to proximal $3-4 \mathrm{~cm}$ ureter, which is the site for majority of shunts.

\section{Conclusion}

MRI is new \& non invasive method for imaging of the thoracic duct, cysterna chyli \& dilated lymphatics. It should be the investigation of choice for patient with 
suspicion of lymphatic obstruction leading to dilatation and lympho-urinary fistula.

\section{REFERENCES}

[1] U. T. Aye and S. T. T. Aung, "Chyluria," Clinical Radiology, Vol. 26, No. 2, 1975, pp. 237-242.

[2] H. Ngan and C. H. Leong, "A Lymphographic Study of Chyluria,” British Journal of Radiology, Vol. 50, No. 600, 1977, pp. 863-870. doi:10.1259/0007-1285-50-600-863

[3] A. K. Hemal and N. P. Gupta, "Retroperitoneoscopic Lymphatic Management of Intractable Chyluria,” Journal of Urology, Vol. 167, No. 6, 2002, pp. 2473-2476. doi:10.1016/S0022-5347(05)65007-0

[4] S. Sharma and A. K. Hemal, "Chyluria-An Overview," Nephro-Urology Monthly, Vol. 1, No. 1, 2009, pp. 14-26.

[5] P. R. Nandy, U. S. Dwivedi, N. Vyas, M. Prasad, B. Dutta and P. B. Singh, "Povidone Iodine and Dextrose Solution Combination Sclerotherapy in Chyluria," Urology, Vol. 64, No. 6, 2004, pp. 1107-1109. doi:10.1016/j.urology.2004.07.035

[6] V. K. Mehta, H. Lohar, G. K. Banerjee, M. V. Reddy and
B. C. Harinath, "Surgical Filariasis: Immunoscreening for Filarial IgG Antibodies Using Wuchereria Bancrofti Microfilarial Excretory-Secretory Antigen,” Journal of Communicable Diseases, Vol. 31, No. 1, 1999, pp. 35-40.

[7] X. Zhang, Z. Q. Ye, Z. Chen, Z. Q. Chen, Q. G. Zhu, M. Xin, et al., "Comparison of Open Surgery versus Retroperitoneoscopic Approach to Chyluria," Journal of Urology, Vol. 169, No. 3, 2003, pp. 991-993. doi:10.1097/01.ju.0000045090.45767.56

[8] S. Govil, A. Justus, R. Lakshminarayanan, S. Nayak, A. Devasia and G. Gopalakrishnan, "Retroperitoneal Lymphatics on CT and MR,” Abdominal Imaging, Vol. 32, No. 1, 2007, pp. 53-55. doi:10.1007/s00261-006-9036-9

[9] A. Geliebter, N. Torbay, E. F. Bracco, S. A. Hashim and T. B. Van Itallie, "Overfeeding with Medium-Chain Triglyceride Diet Results in Diminished Deposition of Fat," American Journal of Clinical Nutrition, Vol. 37, No. 1, 1983, pp. 1-4.

[10] K. N. Panicker, N. Arunachalam, N. P. Kumar, J. Prathibha and S. Sabesan, "Efficacy of DiethylcarbamazineMedicated Salt for Microfilaraemia of Brugia Malayi," National Medical Journal of India, Vol. 10, No. 6, 1997, pp. 275-276. 